\title{
Toward Individualized Approaches to Partial Nephrectomy: Assessing the Correlation Between Ischemia Time and Patient Health Status (RECORD2 Project)
}

\author{
Carlo Andrea Bravi ${ }^{a}$, Andrea Mari $^{b}$, Alessandro Larcher ${ }^{a}$, Daniele Amparore $^{c}$, \\ Alessandro Antonelli ${ }^{d}$, Walter Artibani ${ }^{e}$, Roberto Bertini $^{a}$, Pierluigi Bove $^{f}$, \\ Eugenio Brunocilla $^{g, h}$, Luigi Da Pozzo ${ }^{i}$, Fabrizio Di Maida ${ }^{b}$, Cristian Fiori $^{c}$, Andrea Gallioli $^{j}$, \\ Paolo Gontero $^{k}$, Vincenzo Li Marzi ${ }^{l}$, Nicola Longo ${ }^{m}$, Vincenzo Mirone ${ }^{m}$, Francesco Porpiglia ${ }^{c}$, \\ Bernardo Rocco $^{n}$, Riccardo Schiavina ${ }^{g, h}$, Luigi Schips $^{o}$, Claudio Simeone ${ }^{d}$, \\ Salvatore Siracusano $^{e}$, Riccardo Tellini $^{b}$, Carlo Terrone $^{p}$, Carlo Trombetta $^{q}$, Vincenzo Ficarra ${ }^{r}$, \\ Marco Carini $^{b}$, Francesco Montorsi $^{a}$, Umberto Capitanio $^{a}$, Andrea Minervini ${ }^{b, *}$, \\ Contributors: Vincenzo Altieri Francesco Berardinelli Marco Borghesi Antonio Celia Luca \\ Cindolo Elisabetta Costantini Julian Daja Mario Falsaperla Maria Furlan Angelo Porreca Marco \\ Roscigno Cesare Selli Alchiede Simonato Giuseppe Vespasiani Donata Villari Alessandro Volpe.
}

\footnotetext{
${ }^{a}$ Division of Oncology/Unit of Urology, URI-Urological Research Institute, Vita-Salute University, IRCCS San Raffaele Hospital, Milan, Italy; ${ }^{\mathrm{b}}$ Department of Urology, University of Florence, Unit of oncologic minimally-Invasive Urology and Andrology, Careggi Hospital, Florence, Italy; ${ }^{\mathrm{C}}$ Division of Urology, Department of Oncology, San Luigi Gonzaga Hospital, School of Medicine, Orbassano, Turin, Italy; ${ }^{\mathrm{d}}$ Department of Urology, Ospedali Civili Hospital, University of Brescia, Brescia, Italy; ${ }^{\mathrm{e}}$ Department of Urology, Azienda Ospedaliera Universitaria Integrata (A.O.U.I.), Verona, Italy; ${ }^{\mathrm{f}}$ Department of Urology, University Hospital of Tor Vergata, Rome, Italy; ${ }^{\mathrm{g}}$ Department of Urology, University of Bologna, Bologna, Italy; ${ }^{\mathrm{h}}$ Department of Experimental, Diagnostic, and Specialty Medicine, University of Bologna, Bologna, Italy; ${ }^{\mathrm{i}}$ Department of Urology, Papa Giovanni XXIII Hospital, Bergamo, Italy; ${ }^{j}$ Department of Urology, Fondazione IRCCS Ca' Granda, Ospedale Maggiore Policlinico, University of Milan, Milan, Italy; ${ }^{\mathrm{k}}$ Division of Urology, Department of Surgical Sciences, San Giovanni Battista Hospital, University of Turin, Turin, Italy; ${ }^{1}$ Department of Urology, Unit of Urological Minimally Invasive Robotic Surgery and Renal Transplantation, Careggi Hospital, University of Florence, Florence, Italy; ${ }^{\mathrm{m}}$ Department of Urology, University Federico II, Naples, Italy; ${ }^{\mathrm{n}}$ Department of Urology, Ospedale Policlinico e Nuovo Ospedale Civile S. Agostino Estense Modena, University of Modena and Reggio Emilia, Modena, Italy; ${ }^{\circ}$ Department of Urology, SS Hospital. Annunziata, Chieti, Italy; ${ }^{\mathrm{p}}$ Department of Urology, University of Genova, Genova, Italy; ${ }^{\mathrm{q}}$ U.C.O. Clinica Urologica, Università degli Studi di Trieste, Trieste, Italy; ${ }^{\mathrm{r}}$ Department of Human and Paediatric Pathology, Gaetano Barresi, Urologic Section, University of Messina, Messina, Italy
}

Article info

Article history:

Received 8 January 2020

Received in revised form

13 May 2020

Accepted May 20, 2020

Associate Editor:

Alberto Briganti

\begin{abstract}
Background: Ischemia time during partial nephrectomy (PN) is among the greatest determinants of acute kidney injury (AKI). Whether this association is affected by the preoperative risk of AKI has never been investigated.

Objective: To assess the effect of the interaction between the preoperative risk of AKI and ischemia time on the probability of AKI during PN.

Design, setting, and participants: Data of 944 patients treated with on-clamp PN for cT1 renal tumors were extracted from the Registry of Conservative and Radical Surgery for Cortical Renal Tumor Disease (RECORD2) database, a prospective multicenter project.

Outcome measurements and statistical analysis: We estimated the preoperative risk of AKI (defined according to the risk/injury/failure/loss/end-stage [RIFLE]

\footnotetext{
* Corresponding author. Department of Urology, Careggi Hospital, University of Florence, Florence, Italy. Tel.: +390552758011; Fax: +390552758014.

E-mail address: andreamine@libero.it (A. Minervini).
}

https://doi.org/10.1016/j.euo.2020.05.009

2588-9311/@ 2020 European Association of Urology. Published by Elsevier B.V. All rights reserved. 


\section{Keywords:}

Renal cell carcinoma

Partial nephrectomy

Nephron-sparing surgery

Warm ischemia

Renal function

Acute kidney injury

Multimodal approach

Preoperative counseling criteria) according to age, baseline renal function, clinical stage, preoperative aspects and dimensions used for an anatomical (PADUA) score, and surgical approach. Classification and regression tree (CART) analysis identified patients at "high" and "low" risk of AKI. Finally, we plotted the probability of AKI over ischemia time stratified by the preoperative risk of AKI.

Results and limitations: Overall, 235 (25\%) patients experienced AKI after surgery. At multivariable analysis, older patients, those with more complex tumors, those with higher baseline function, and those treated with open surgery had an increased risk of AKI (all $p \leq 0.011$ ). According to the first split at CART analysis, patients were categorized as those with "high" and "low" risk of AKI having a probability of $>40 \%$ or $<40 \%$. For low-risk patients, the probability of AKI in case of $<10$ versus $>20 \mathrm{~min}$ of ischemia was $13 \%$ versus $28 \%$ (absolute risk increase $15 \%$ ). The risk of AKI for highrisk patients who had $<10$ versus $>20 \mathrm{~min}$ of ischemia was $31 \%$ versus $77 \%$. This corresponds to an absolute risk increase of $45 \%$. Limitations include retrospective data analyses and lack of surgeons' prior experience.

Conclusions: Ischemia time during PN has different implications for patients with different health status. Clamp time seems less clinically relevant for patients in good conditions who may endure prolonged ischemia with a mild increase in the risk of AKI, whereas frail patients seem to be more vulnerable to ischemic damage even for short clamp time. For individualized intra- and postoperative management, duration of ischemia needs to be questioned in the context of the individual health status. Patient summary: Functional sequelae related to ischemia time during partial nephrectomy depend on baseline health status. The correlation between the duration of ischemia and baseline health status should be taken into account toward individualized intra- and postoperative management.

(c) 2020 European Association of Urology. Published by Elsevier B.V. All rights reserved.

\section{Introduction}

Acute kidney injury (AKI) occurs in approximately 20\% of partial nephrectomies [1-3]. It has been established that AKI affects long-term renal function negatively [4,5], and consequently, it has relevant implications in survival and quality of life after surgery [6,7]. As such, many efforts have been made to identify causal factors and predictors of AKI to prevent its occurrence during surgery [8-11].

Ischemia time is among the greatest determinants of AKI, and it is widely accepted that a warm ischemia shorter than 20-25 min might avoid functional consequences [12]. However, evidence of AKI after clampless procedures [13] implies that other factors are involved in acute damage during partial nephrectomy. This makes a strong argument toward a multifactorial etiology of AKI, suggesting that both the duration of ischemia and the individual risk contribute to functional damage. However, the relative contribution of each of these factors to the risk of AKI has never been investigated. In this regard, it seems reasonable that ischemia might have different implications according to the individual health profile, that is, the same clamp time might be more harmful for an 80 -yr-old patient with several comorbidities than for a healthy 50-yr-old individual. This may be extremely relevant for surgical planning, but unfortunately current literature on this topic is limited. For this reason, we here sought to examine the relative contribution of individual risk and ischemia time to the probability of AKI after partial nephrectomy in a large multiinstitutional series.

\section{Patients and methods}

The Italian Registry of Conservative and Radical Surgery for Cortical Renal Tumor Disease (RECORD2 project) is a prospective, observational project promoted by the Italian Society of Urology, the collection criteria of which were described previously [14].

We analyzed the data of 2314 patients diagnosed with a cT1 NO MO renal mass at computed tomography or magnetic resonance imaging, and treated with partial nephrectomy from 2013 to 2016. For the scope of this study, we focused on on-clamp procedures $(n=1235)$. The surgical approach was selected according to surgeon's choice. To mitigate confounding by the established relationship between ischemia time and tumor complexity, we excluded patients with preoperative aspects and dimensions used for an anatomical (PADUA) score [15] $\geq 10$ tumors $(n=230)$. Patients with solitary kidney $(n=8)$, those who received cold ischemia $(n=44)$, and those with missing covariates (age, $n=4$; preoperative renal function, $n=5$ ) were also excluded, resulting in 944 patients eligible for analysis.

Our primary aim was to investigate whether the impact of warm ischemia on the risk of acute injury might be affected by the individual preoperative risk of AKI. AKI was defined according to the risk/injury/ failure/loss/end-stage (RIFLE) criteria [16] using postoperative renal function up to the 3rd postoperative day.

Our statistical analysis involved four steps. First, differences in baseline characteristics between patients who had AKI and those who did not were assessed using the Kruskal-Wallis and chi-square tests. For descriptive purposes, we included all the patients regardless of the severity of the injury. To mitigate confounding, subsequent analyses did not include patients who had stage 2-3 AKI $(n=24)$. Second, we used a multivariable logistic regression (model 1 ) to calculate the individual preoperative risk of AKI. Covariates consisted of age, clinical T stage (T1a vs T1b), preoperative estimated glomerular function rate (eGFR), total PADUA score [15] (continuous), and surgical approach (open vs 
laparoscopic vs robotic). Assuming that the planned surgical technique was confirmed after surgery, we also explored the inclusion of the type of resection (enucleation vs enucleoresection [17]) as a proxy of preserved parenchyma (model 2; Supplementary Table 1). Since data from different institutions are correlated, we incorporated institution clustering in our analysis using the cluster option in Stata statistical software. The model was corrected for overfit using 10 -fold cross validation. To assess the relationship between preoperative risk and ischemia time (ie, that the distribution of ischemia time was homogeneous regardless of the preoperative risk), we used a nonparametric LOWESS function (Supplementary Fig. 1). Calibration plot analyses assessed the accuracy of the model (Supplementary Fig. 2). Third, the individual probability of AKI derived from model 1 was used as the independent variable for classification and regression tree (CART) analysis in an effort to identify the most meaningful cutoff(s) for the identification of patients who will and will not experience AKI after surgery. According to the first split at CART analysis, we then stratified patients into groups of "high" and "low" risk of AKI. Finally, to visualize our findings, we plotted the preoperative probability of AKI over the duration of ischemia stratified by preoperative risk using a nonparametric curve fitting method.

All analyses were conducted using Stata 14 (Stata Corp., College Station, TX, USA).

\section{Results}

The descriptive characteristics of our cohort are described in Table 1. Overall, 235 (25\%) patients had postoperative AKI. Acute injury was more frequent for older patients, those treated with open surgery, and those who had more complex tumors. Ischemia during surgery was longer among patients who experienced postoperative AKI.

Table 2 describes our preoperative model to predict AKI. At multivariable analysis, age (odds ratio [OR]: $1.03 ; 95 \%$ confidence interval [95\% CI]: 1.02, 1.05; $p<0.0001)$, preoperative eGFR (OR: 1.02; 95\% CI: 1.01, 1.03; $p=0.003$ ), clinical T1b stage (OR: 1.88; 95\% CI: 1.35, 2.62; $p=0.0002$ ), and higher PADUA score (OR: 1.20; 95\% CI: 1.05, 1.37; $p=0.007$ ) were associated with an increased risk of AKI. Conversely, laparoscopic (OR: 0.47; 95\% CI: 0.26, 0.84; $p=0.011$ ) and robotic (OR: 0.39; 95\% CI: 0.25, 0.60; $p<0.0001$ ) surgery had a lower probability of AKI than open surgery. After cross validation, the discrimination accuracy of this model (area under the curve) was 67\% (95\% CI: $63 \%, 71 \%$ ) in our cohort.

The individual nomogram-derived probability of AKI was used as the independent variable for CART analysis. According to the first split at CART analysis, patients were considered to be at a "high" risk of AKI if their preoperative probability was $>40 \%$; patients who had a baseline probability of $<40 \%$ were considered to be at a "low" risk.

Figure 1 shows the observed likelihood of AKI over warm ischemia time. We can see that the relationship between the duration of ischemia and probability of AKI was different according to the preoperative risk of AKI. As an example, in case of $<10$ versus $>20$ min of ischemia, low-risk patients had a risk of AKI of $13 \%$ (95\% CI: $10 \%, 17 \%)$ versus $28 \%(95 \%$ CI: 22\%, 34\%) (absolute risk increase 15\%; 95\% CI: 7\%, 22\%). By contrast, the risk of AKI for high-risk patients who had $<10$ versus $>20$ min of ischemia was $31 \%$ (95\% CI: $17 \%, 51 \%$ ) versus $77 \%$ (95\% CI: $63 \%, 89 \%)$. This corresponds to an absolute risk increase of $45 \%$ (95\% CI: $19 \%, 68 \%$ ).
Table 1 - Descriptive characteristics of 944 patients treated with on-clamp partial nephrectomy for $\mathrm{T} 1$ renal tumor.

\begin{tabular}{|c|c|c|c|}
\hline & $\begin{array}{c}\text { No AKI } \\
(N=709 ; 75 \%)\end{array}$ & $\begin{array}{c}\text { AKI } \\
(N=235 ; 25 \%)\end{array}$ & $p$ value \\
\hline Age (yr) & $64(54,72)$ & $66(59,73)$ & 0.002 \\
\hline BMI $(N=940)$ & $26(23,28)$ & $26(24,28)$ & 0.2 \\
\hline \multicolumn{4}{|l|}{ Sex } \\
\hline Male & $433(61 \%)$ & $166(71 \%)$ & 0.008 \\
\hline Female & $276(39 \%)$ & $69(29 \%)$ & \\
\hline Preoperative eGFR & $87(72,101)$ & $88(75,103)$ & 0.11 \\
\hline Hypertension & $71(10 \%)$ & $31(13 \%)$ & 0.2 \\
\hline Diabetes & $43(6 \%)$ & $9(4 \%)$ & 0.2 \\
\hline \multicolumn{4}{|c|}{ Charlson Comorbidity Index } \\
\hline $0-1$ & $97(14 \%)$ & $19(8 \%)$ & 0.068 \\
\hline $2-4$ & $394(56 \%)$ & $143(61 \%)$ & \\
\hline $5+$ & $218(31 \%)$ & $73(31 \%)$ & \\
\hline \multicolumn{4}{|l|}{ ECOG score } \\
\hline $0-1$ & $678(96 \%)$ & $221(94 \%)$ & 0.6 \\
\hline 2 & $29(4 \%)$ & $13(5 \%)$ & \\
\hline $3+$ & $2(<1 \%)$ & $1(1 \%)$ & \\
\hline \multicolumn{4}{|l|}{ ASA score } \\
\hline 1 & $129(18 \%)$ & $32(14 \%)$ & 0.12 \\
\hline 2 & $457(64 \%)$ & $152(65 \%)$ & \\
\hline $3+$ & $121(17 \%)$ & $51(22 \%)$ & \\
\hline Unknown & $2(<1 \%)$ & 0 & \\
\hline \multicolumn{4}{|l|}{ Side of lesion } \\
\hline Right & $361(51 \%)$ & $135(57 \%)$ & 0.082 \\
\hline Left & $348(49 \%)$ & $100(43 \%)$ & \\
\hline \multicolumn{4}{|l|}{ Clinical T stage } \\
\hline T1a & $563(79 \%)$ & $155(66 \%)$ & $<0.0001$ \\
\hline T1b & $146(21 \%)$ & $80(34 \%)$ & \\
\hline \multicolumn{4}{|l|}{ PADUA score } \\
\hline 6 & $176(25 \%)$ & $35(15 \%)$ & 0.005 \\
\hline $7-8$ & $404(57 \%)$ & $146(62 \%)$ & \\
\hline 9 & $129(18 \%)$ & $54(23 \%)$ & \\
\hline \multicolumn{4}{|l|}{ Surgical approach } \\
\hline Open & $133(19 \%)$ & $84(36 \%)$ & $<0.0001$ \\
\hline Laparoscopic & $204(29 \%)$ & $56(24 \%)$ & \\
\hline Robotic & $372(52 \%)$ & $95(40 \%)$ & \\
\hline Ischemia time (min) & $15(11,19)$ & $18(14,22)$ & $<0.0001$ \\
\hline \multicolumn{4}{|c|}{$\begin{array}{l}\text { AKI = acute kidney injury; ASA = American Society of. Anesthesiologists; } \\
\text { BMI = body mass index; ECOG = Eastern Cooperative Oncology Group; } \\
\text { eGFR = estimated glomerular function rate; PADUA = preoperative aspects } \\
\text { and dimensions used for an anatomical. } \\
\text { Data are presented as medians and interquartile ranges, and as frequencies } \\
\text { and proportions for continuous and categorical variables, respectively. The } \\
\text { number in parenthesis represents the number of patients with available } \\
\text { data. }\end{array}$} \\
\hline
\end{tabular}

Table 2 - Multivariable logistic regression for the prediction of postoperative AKI in 920 patients treated with partial nephrectomy for $\mathrm{T} 1$ renal tumor.

\begin{tabular}{llll}
\hline & Odds ratio & $\begin{array}{l}\text { 95\% Confidence } \\
\text { interval }\end{array}$ & $p$ value \\
\hline Age & 1.03 & $1.02,1.05$ & $<0.0001$ \\
\hline Clinical T stage & Ref & & \\
$\quad$ T1a & 1.88 & $1.35,2.62$ & 0.0002 \\
$\quad$ T1b & 1.20 & $1.05,1.37$ & 0.007 \\
\hline PADUA score & 1.02 & $1.01,1.03$ & 0.003 \\
\hline $\begin{array}{l}\text { Preoperative eGFR } \\
\text { Surgical approach }\end{array}$ & Ref & & \\
$\quad$ Open & 0.47 & $0.26,0.84$ & 0.011 \\
$\quad$ Laparoscopic & 0.39 & $0.25,0.60$ & $<0.0001$ \\
\hline Robotic & & & \\
\hline
\end{tabular}

AKI = acute kidney injury; eGFR=estimated glomerular function rate; PADUA = preoperative aspects and dimensions used for an anatomical. 


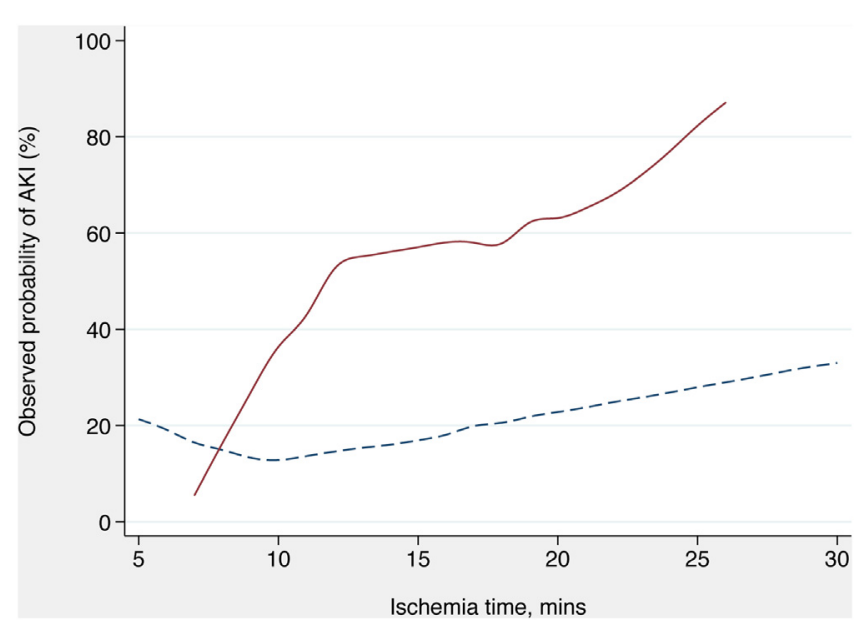

Fig. 1 - Relationship between observed AKI and ischemia time stratified by preoperative risk of AKI. The red line represents preoperative risk $>40 \%$ and the dashed line represents preoperative risk $\leq 40 \%$. AKI = acute kidney injury.

\section{Discussion}

We noted that the functional damage related to ischemia seems to be related to the probability of having AKI estimated before surgery. In other words, the same duration of ischemia might have different implications according to the individual risk of functional harm.

Ever since partial nephrectomy has gained popularity, considerable research has focused on determining the safety threshold for intraoperative ischemia time. According to a recent systematic review [12], a 20-25 min cutoff can fairly separate patients who develop and do not develop functional decline after partial nephrectomy. This is important to assess the general contribution of ischemia to functional damage, but the adoption of a specific threshold may be problematic on an individual basis. In a typical study, the association between outcome and ischemia is investigated in the overall cohort after adjusting for confounding. This raises two critical issues. First, stating that an ischemia shorter than 20 min will be without consequences might not be correct for all the patients. Surgical planning cannot rely on the one-size-fits-all approach but should take into account individual characteristics. In the era of individualized surgery, it seems remarkable that we focus on postoperative features such as preserved parenchyma, but virtually nothing looking at the preoperative risk of functional loss. Our findings suggest that ischemia time cannot be considered an absolute value, but rather that the right surgery should be given to the right patient according to the risk of functional harm. This entails a second critical point, that is, a clear need for the standardization of functional profile before, during, and after renal surgery. While tumor complexity guides indication for surgery (eg, radical vs partial nephrectomy), we have to bear in mind that the final goal of partial nephrectomy is to preserve renal function, and as such, an appropriate estimation of functional risk is necessary to optimize ischemia time. Similarly, any given duration of ischemia should be considered in light of individual health status if personalized management after surgery is contemplated, with the final goal of referring patients at a high risk of functional deterioration to a tailored, multidisciplinary evaluation. Although the general recommendation toward shortening ischemia time still holds true [12], surgeons should consider a more granular approach based on the assessment of functional profile. In this context, our study represents a benchmark to implement the assessment of functional risk in future investigations.

It is noteworthy that we did not include comorbidities in our preoperative model. Although prior investigators observed an association between AKI and diabetes [18], hypertension [10,19], or smoking status [10], there are reasons to believe that this relationship might be more apparent than real. First, other papers failed to confirm these findings [10,20,21], suggesting that the established association between medical conditions and renal function [5] might not hold true when taking into account a one-time event such as surgical AKI. Among possible explanations, the majority of studies on this issue included patients with solitary kidney in whom adaptive phenomena might influence functional profile, particularly in case of AKI. Therefore, it is plausible that the same conclusions may not be applicable to patients with two functional units $[4,22,23]$. Moreover, patients are usually classified as those having a condition even if they are on drug therapy and measurement parameters have normalized at the time of surgery. It is thus unclear how we should consider, say, a patient with hypertension that is perfectly controlled by medical therapy. Since there is evidence that microvascular changes related to high blood pressure are reversible [24], it is reasonable that hypertension might not affect the agerelated renal function of such patient. For these reasons, we are confident that the relative contribution of comorbidities to renal function is reflected into age and preoperative eGFR, which are included in our risk estimation.

Our study is not devoid of limitations. Although we adjusted for patient and tumor characteristics, we cannot entirely rule out potential residual confounding from known and unknown variables. This may help explain the association between surgical approach and AKI resulted from our multivariable model. A potential reason might be the lack of surgeon's experience among our covariates. To address this issue, we excluded patients with complex renal masses that might have resulted in longer ischemia for less experienced surgeons. Moreover, we performed sensitivity analysis on patients receiving surgery at institutions with an annual caseload of $>20$ procedures, with no meaningful differences in results (Supplementary Table 2). A second limitation concerns the definition of AKI. There is evidence that new biomarkers or preoperative proteinuria might improve the sensitivity for the detection of subclinical AKI [11]. Accordingly, the lack of such information in our study might have resulted in underestimation of AKI. Moreover, according to a recent consensus definition [25], AKI should be investigated up to $7 \mathrm{~d}$ after surgery, while our dataset included renal function up to the 3rd postoperative day. It 
could thus be hypothesized that some cases of AKI might have been missed. However, there is evidence that the majority of acute injuries occur in the first $3 \mathrm{~d}$ after surgery [4], and therefore, we are confident that the small number of patients who might have contributed to this bias did not affect our results. Finally, we have to acknowledge that our analyses did not include the evaluation of parenchymal mass reduction [26,27], a morphologic measure of renal functional parenchyma. Although such construct was not available for the analyses, our results were not altered in sensitivity analysis, including a proxy of spared parenchy$\mathrm{ma}$, that is, the extension of surgical resection. For these reasons, we are confident that our findings were not a consequence of the lack of parenchymal mass reduction in our analyses.

Our results have implications for empirical research. Evidence that outcomes of surgery are influenced by surgical experience $[28,29]$ suggests that the inclusion of such a feature might improve risk stratification. It would not be surprising that the probability of functional damage might be lowered using the surgical approach a surgeon is more confident with [30]. This suggests that a surgeon's prior experience should be taken into consideration for preoperative counseling. In this regard, valuable standardization of experience is provided by surgical curricula [31]. In addition, our results suggest that more serious attention should be paid to functional profile before partial nephrectomy. Many efforts have been made to identify patients at risk of functional loss $[1,8,9,32-34]$, and it seems so far established that AKI should be prevented as it is associated with long-term function [4,35,36]. A quite generic recommendation is for preoperative correction of medical conditions. This intervention is surely beneficial for patients, but it is unlikely to influence surgical strategy, let alone that chronic comorbidities are difficult to eradicate in few months from diagnosis to surgical treatment. Moreover, there is evidence that acute damage is often related with intraoperative factors such as operative time, blood loss, and ischemia time [8,9], and this is consistent with our finding that surgical approach affects the risk of AKI. For this reason, it seems reasonable to optimize technical aspects of surgery according to the individual risk of AKI, which, in turn, has to be estimated before surgery. Accordingly, systematic research is required to identify critical aspects of partial nephrectomy that are associated with AKI. In addition, other implementations should be investigated. For instance, serum creatinine may be inadequate for gauging side-specific renal function. This is extremely relevant for renal surgery as the affected kidney might be, say, the one less contributing to global function, resulting in a risk/benefit ratio in favor of radical rather than partial nephrectomy. Conversely, more efforts for partial nephrectomy might be considered if surgery was needed in the kidney with the highest contribution to global function. In this regard, imaging modalities such as renal scintigraphy might be of added value for preoperative planning. However, current literature on this issue is limited and mainly focused on the assessment of function after surgery [37]. It is reasonable that the implementation of renal scintigraphy to preoperative counseling might result in more accurate risk stratification, thereby improving surgical planning and likely translating into better surgery. We intend to investigate these possibilities in future studies.

\section{Conclusions}

A given duration of ischemia during partial nephrectomy has different implications for patients with different health status. Clamp time seems to be less clinically relevant for patients in good conditions who may endure prolonged ischemia with a mild increase of AKI risk, whereas frail patients seem to be more vulnerable to ischemia damage and may require additional interventions in the postoperative period. This should be taken into account toward individualized management before and after partial nephrectomy.

Author contributions: Andrea Minervini had full access to all the data in the study and takes responsibility for the integrity of the data and the accuracy of the data analysis.

Study concept and design: Bravi, Bertini, Capitanio, Larcher, Minervini. Acquisition of data: Mari, Bove, di Maida, Amparore, Gallioli, Fiori, Li Marzi, Schips, Siracusano, Trombetta.

Analysis and interpretation of data: Bravi, Larcher, Capitanio, Mari. Drafting of the manuscript: Bravi, Minervini.

Critical revision of the manuscript for important intellectual content: Antonelli, Artibani, Bertini, Brunocilla, Da Pozzo, Gontero, Longo, Mirone, Porpiglia, Schiavina, Tellini, Simeone, Rocco, Terrone, Montorsi, Minervini.

Statistical analysis: Bravi, Larcher, Capitanio, Mari.

Obtaining funding: Minervini, Carini, Ficarra.

Administrative, technical, or material support: None.

Supervision: Artibani, Mirone, Ficarra, Carini, Minervini, Montorsi. Other: None.

Financial disclosures: Andrea Minervini certifies that all conflicts of interest, including specific financial interests and relationships and affiliations relevant to the subject matter or materials discussed in the manuscript (eg, employment/affiliation, grants or funding, consultancies, honoraria, stock ownership or options, expert testimony, royalties, or patents filed, received, or pending), are the following: None.

Funding/Support and role of the sponsor: This work was supported by the Italian Society of Urology (SIU).

\section{Appendix A. Supplementary data}

Supplementary material related to this article can be found, in the online version, at doi:https://doi.org/10.1016/j. euo.2020.05.009.

\section{References}

[1] Martini A, Cumarasamy S, Beksac AT, et al. A nomogram to predict significant estimated glomerular filtration rate reduction after robotic partial nephrectomy. Eur Urol 2018;74:833-9.

[2] Capitanio U, Bensalah K, Bex A, et al. Epidemiology of renal cell carcinoma. Eur Urol 2019;75:74-84. 
[3] Capitanio U, Montorsi F. Renal cancer. Lancet 2016;387:894-906.

[4] Bravi CA, Vertosick E, Benfante N, et al. Impact of acute kidney injury and its duration on long-term renal function after partial nephrectomy. Eur Urol 2019;76:398-403.

[5] Chawla LS, Eggers PW, Star RA, Kimmel PL. Acute kidney injury and chronic kidney disease as interconnected syndromes. N Engl J Med 2014;371:58-66.

[6] Capitanio U, Terrone C, Antonelli A, et al. Nephron-sparing techniques independently decrease the risk of cardiovascular events relative to radical nephrectomy in patients with a T1a-T1b renal mass and normal preoperative renal function. Eur Urol 2015;67 (4):683-9. http://dx.doi.org/10.1016/j.eururo.2014.09.027.

[7] Antonelli A, Minervini A, Sandri M, et al. Below safety limits, every unit of glomerular filtration rate counts: assessing the relationship between renal function and cancer-specific mortality in renal cell carcinoma. Eur Urol 2018;74:661-7.

[8] Martini A, Sfakianos J, Paulucci D, et al. Predicting acute kidney injury after robot-assisted partial nephrectomy: implications for patient selection and postoperative management. Urol Oncol 2019;37:445-51.

[9] Bertolo R, Garisto J, Li J, Dagenais J, Kaouk J. Development and internal validation of a nomogram for predicting renal function after partial nephrectomy. Eur Urol Oncol 2019;2:106-9.

[10] Rajan S, Babazade R, Govindarajan SR, et al. Perioperative factors associated with acute kidney injury after partial nephrectomy. Br J Anaesth 2016;116:70-6.

[11] Antonelli A, Allinovi M, Cocci A, et al. The predictive role of biomarkers for the detection of acute kidney injury after partial or radical nephrectomy: a systematic review of the literature. Eur Urol Focus 2020;6:344-53.

[12] Volpe A, Blute ML, Ficarra V, et al. Renal ischemia and function after partial nephrectomy: a collaborative review of the literature. Eur Urol 2015;68(February):61-74.

[13] Kawamura N, Yokoyama M, Tanaka H, et al. Acute kidney injury and intermediate-term renal function after clampless partial nephrectomy. Int J Urol 2018;65:372-6.

[14] Mari A, Campi R, Schiavina R. Nomogram for predicting the likelihood of postoperative surgical complications in patients treated with partial nephrectomy: a prospective multicenter observational study (the RECORd 2 project). BJU Int 2019;124:93-102.

[15] Ficarra V, Novara G, Secco S, et al. Preoperative aspects and dimensions used for an anatomical (PADUA) classification of renal tumours in patients who are candidates for nephron-sparing surgery. Eur Urol 2009;56:786-93.

[16] Bellomo R, Ronco C, Kellum J, Mehta R, Palevsky P. Acute renal failure-definition, outcome measures, animal models, fluid therapy and information technology needs: the Second International Consensus Conference of the Acute Dialysis Quality Initiative (ADQI) group. Crit Care 2004;8:R204-9.

[17] Minervini A, Carini M, Uzzo RG, Campi R, Smaldone MC, Kutikov A. Standardized reporting of resection technique during nephronsparing surgery: the surface-intermediate-base margin score. Eur Urol 2014;66:803-5.

[18] Zabell J, Isharwal S, Dong W, et al. Acute kidney injury after partial nephrectomy of solitary kidneys: impact on long-term stability of renal function. J Urol 2018;200:1295-301.

[19] Capitanio U, Larcher A, Cianflone F, et al. Hypertension and cardiovascular morbidity following surgery for kidney cancer. Eur Urol Oncol 2020;3:209-15.

[20] Beksac AT, Reddy BN, Martini A, et al. Hypertension and diabetes mellitus are not associated with worse renal functional outcome after partial nephrectomy in patients with normal baseline kidney function. Int J Urol 2018;26:120-5.

[21] Isharwal S, Ye W, Wang A, et al. Impact of comorbidities on functional recovery from partial nephrectomy. J Urol 2018;199:1433-9.

[22] Bravi CA. Reply to Won Ho Kim, Hyun-Kyu Yoon, Chang Wook Jeong's Letter to Editor re: Carlo Bravi, Emily Vertosick, Nicole Benfante, et al. Impact of acute kidney injury, its duration on long-term renal function after partial nephrectomy. Eur Urol 2019;76:398-403, Eur Urol 2020;77:e16-7.

[23] Bertolo R, Capitanio U. Re: Acute kidney injury after partial nephrectomy in solitary kidneys: impact on long-term stability of renal function. Eur Urol 2019;75:346-8.

[24] Cameron AC, Lang NN, Touyz RM. Drug treatment of hypertension: focus on vascular health. Drugs 2016;76:1529-50.

[25] Chawla LS, Bellomo R, Bihorac A, et al. Acute kidney disease and renal recovery: consensus report of the Acute Disease Quality Initiative (ADQI) 16 Workgroup. Nat Rev Nephrol 2017;13:241-57.

[26] Zhang Z, Zhao J, Dong W, et al. Acute ipsilateral renal dysfunction after partial nephrectomy in patients with a contralateral kidney: spectrum score to unmask ischemic injury. Eur Urol 2016;70:692-8.

[27] Zhang Z, Zhao J, Dong W, et al. Acute kidney injury after partial nephrectomy: role of parenchymal mass reduction and ischemia and impact on subsequent functional recovery. Eur Urol 2016;69:745-52.

[28] Bravi CA, Tin A, Vertosick E, et al. The impact of experience on the risk of surgical margins and biochemical recurrence after robotassisted radical prostatectomy: a learning curve study. J Urol 2019;202:108-13.

[29] Larcher A, Muttin F, Peyronnet B, et al. The learning curve for robotassisted partial nephrectomy: impact of surgical experience on perioperative outcomes. Eur Urol 2019;75:253-6.

[30] Larcher A, Capitanio U, De Naeyer G, et al. Is Robot-assisted surgery contraindicated in the case of partial nephrectomy for complex tumours or relevant comorbidities? A comparative analysis of morbidity, renal function, and oncologic outcomes. Eur Urol Oncol 2018;1:61-8

[31] Larcher A, De Naeyer G, Turri F, et al. The ERUS curriculum for robotassisted partial nephrectomy: structure definition and pilot clinical validation. Eur Urol 2019;75:1023-31.

[32] Bravi CA, Larcher A, Capitanio U, et al. Perioperative outcomes of open, laparoscopic, and robotic partial nephrectomy: a prospective multicenter observational study (the RECORd 2 project). Eur Urol Focus. In press. https://doi.org/10.1016/j.euf.2019.10.013.

[33] Martini A, Falagario Ug, Cumarasamy S, et al. Defining risk categories for a significant decline in estimated glomerular filtration rate after robotic partial nephrectomy: implications for patient followup. Eur Urol Oncol 2021;4:498-501.

[34] Capitanio U, Larcher A, Kriegmair MC, et al. Do we truly care about the functional outcomes for renal cancer patients? Multidisciplinarity is still far away. Eur Urol 2019;75:349-50.

[35] Bravi CA, Vickers A. Why acute kidney injury during partial nephrectomy matters. Ann Transl Med 2019;7:S317.

[36] Porpiglia F, Amparore D, Checcucci E, Fiori C. Parenchymal mass preserved after partial nephrectomy and "global renal damage": two faces of the same coin. Eur Urol Oncol 2019;2:104-5.

[37] Sankin A, Sfakianos JP, Schiff J, Sjoberg D, Coleman JA. Assessing renal function after partial nephrectomy using renal nuclear scintigraphy and estimated glomerular filtration rate. Urology 2012;80:343-6. 